\title{
Reflections on palliative care
}

\author{
Keywords: child, adult, elderly, death, family
}

\section{Opinion}

Whether the patient is a child, adult or elderly in the process of death, implies having a good listening and being able to direct the assistance in order to collect important information at the right time, give voice to the individual and freedom that extends the family, letting them vent their anxieties and uncertainties in the face of the nearness or possibility of death. Palliate is protect. The equivocal relationship between palliative care and euthanasia amputates the holistic notion of this type of reception that includes, in addition to physical symptoms, social, spiritual and psychological issues that may arise as a need for sick individuals and their family. A patient with life threatening illness needs palliative care. Due to the severity of this illness, this team must be multiprofessional to be able to attend to the real needs of this patient and prioritize them. The main objective of palliative care is to improve the quality of life of the patient and his/ her family.

Health professionals are very close to the terminally ill, but few are capable of listening and generating a therapeutic climate at this delicate time. Several studies that seek to understand the perception of health professionals about palliative care prove the technical lack of preparation of the team; ignorance about the concept and indication of palliative care and uncertainties in the face of eminent death. These fragilities establish in the environment of assistance the insecurity that is aggravated by the lack of psychotherapeutic support of the multiprofessional team that suffers daily with the patient in the process of dying.

In order to provide palliative care to patients, it is essential not only for scientific knowledge about the stages of death; the process of death and dying; procedures and techniques for the relief of pain and suffering as well as investing in the process of self-knowledge. It is necessary to reflect on the possibility of finitude of life, on how we can alleviate suffering, deal with the intentional withdrawal and the uncertainties that the process of dying adds to the patient.

Beyond physical symptoms such as pain and dyspnea, the high emotional, social and spiritual costs of patients undergoing palliative care must also be considered. Concentrating these patients in hospitals, receiving curative assistance, using invasive methods and cutting-edge technology does not always mean ransom to dignity. This therapeutic obstinacy restricts care and does not guarantee improvement in the patient's quality of life.

The posited reflection is that we must guide the palliative care for
Volume I Issue 6 - 2017

\author{
Cynthia Carolina Duarte Andrade,' Luzimar \\ Rangel Moreira ${ }^{2}$ \\ 'Master in Medicine and Assistance from the School of \\ Pharmacy, Federal University of Minas Gerais (UFMG), Brazil \\ ${ }^{2}$ Assistant Professor, Pontifical Catholic University of Minas \\ Gerais, Brazil
}

\begin{abstract}
Correspondence: Cynthia Carolina Duarte Andrade, Master in Medicine and Assistance from the School of Pharmacy of the Federal University of Minas Gerais (UFMG), Av. do ontorno, 9494, Prado. CEP 30I I0-064 - Belo Horizonte, MG, Brazil, Email cynthiacarolinaduarteandrade@gmail.com
\end{abstract}

Received: October 31, 2017 | Published: November 09, 2017

the relief of the suffering of all order, with a sensitive listening of all the causes of pain that this person possessed. Can we approach families, release visitors, authorize food, pets, flowers, confessions of pain that go beyond the physical aspect, without this being considered a personal violation of the routines and standards of the hospital environment? We must think of palliative care as an institution of holistic care, in which the entire health team is focused on the same principles and with the common goal of generating comfort and reducing pain and suffering in the process of dying.

Efforts should be invested to achieve the understanding of death as natural and quality of life as the primary clinical goal. The family should be cared for as much as the patient as they share common pains. Patients and family members should receive accurate information from the health team through active communication about the condition and the approach alternatives so that they can make decisions regarding their moral and cultural values. Death is no milestone to finalize care should be incorporated care during the grieving process of family members. The patient and his family are responsible for always being the focus.

\section{Acknowledgements}

None.

\section{Conflict of interest}

The author declares no conflict of interest. 